\title{
L'évaluation des enseignants au Mexique : entre tentatives de rationalisation administrative et permanence du corporatisme
}

\section{Pablo Cussac}

\section{CpenEdition}

\section{Journals}

Édition électronique

URL : https://journals.openedition.org/ries/8586

DOI : 10.4000/ries.8586

ISSN : 2261-4265

Éditeur

France Education international

Édition imprimée

Date de publication : 1 septembre 2019

Pagination : 19-22

ISBN : 978-2-85420-624-1

ISSN : $1254-4590$

Référence électronique

Pablo Cussac, «L'évaluation des enseignants au Mexique : entre tentatives de rationalisation administrative et permanence du corporatisme », Revue internationale d'éducation de Sèvres [En ligne], 81 | septembre 2019, mis en ligne le 01 septembre 2021, consulté le 13 octobre 2021. URL : http:// journals.openedition.org/ries/8586 ; DOI : https://doi.org/10.4000/ries.8586

Ce document a été généré automatiquement le 13 octobre 2021

(c) Tous droits réservés 


\title{
L'évaluation des enseignants au Mexique : entre tentatives de rationalisation administrative et permanence du corporatisme
}

\author{
Pablo Cussac
}

L'évaluation des enseignants est devenue le principal sujet de la politique éducative mexicaine. Depuis les années 1990, les gouvernements successifs ont tenté de rationnaliser la gestion et les pratiques des enseignants à travers différents instruments d'évaluation. Le Service professionnel enseignant (SPD) mis en place en 2013 est l'aboutissement de ces tentatives de gestion. En introduisant des concours d'opposition et de promotion ainsi que des évaluations quatriennales de la performance, le SPD prétend évaluer la qualité des enseignants mexicains, tout en balisant l'influence syndicale sur la gestion des postes et des promotions. La réforme éducative lancée par López Obrador, élu à la fin de 2018, supprime ce dispositif d'évaluation et ouvre la porte au retour à la situation ex ante, interrogeant ainsi la permanence de l'organisation corporatiste de l'éducation mexicaine.

\section{Le contexte : l'évaluation contre le corporatisme}

2 Comprendre les enjeux de l'évaluation demande un détour par l'histoire corporatiste du système éducatif mexicain. Depuis sa création en 1943, le Syndicat national des travailleurs de l'éducation (SNTE) a été intimement lié au Parti révolutionnaire institutionnel (PRI), qui gouverne le pays entre 1929 et 2000. Dans ce "pacte corporatiste", le syndicat est chargé de nombreuses tâches administratives et politiques, qui vont de la gestion quotidienne des écoles et des enseignants jusqu'à la mobilisation de ses membres lors des élections, en passant par l'attribution des nouveaux postes et promotions. Le rôle du SNTE a été progressivement critiqué au sein du secrétariat de l'éducation publique, des milieux universitaires et du propre syndicat 
- ce qui a donné naissance au courant dissident interne, la Coordination nationale des travailleurs de l'éducation (CNTE) - sans que des changements fondamentaux affectent le pacte.

3 En ce qui concerne l'évaluation des enseignants, celle-ci était traditionnellement réalisée par les directeurs et les superviseurs des écoles et se concentrait essentiellement sur des aspects administratifs. Le tournant des années 1990 marque l'apparition d'une double préoccupation pour l'efficience et les salaires enseignants ${ }^{1}$, ce pourquoi le gouvernement de Salinas de Gortiari négocie avec le SNTE l'introduction du programme "Carrière enseignante ", en 1992. Ce système d'évaluations volontaires permet aux enseignants d'obtenir des compléments de salaire (jusqu'à $+217 \%$ ) en fonction de leur performance à des évaluations définies par des commissions paritaires. S'il reçoit un accueil globalement positif de la part des enseignants, qui y voient une façon non contraignante d'améliorer leurs salaires, au cours des années 2000, les experts éducatifs critiquent l'inefficacité du programme et la perversion des épreuves de connaissances des élèves, qui en 2011 sont utilisées pour déterminer $50 \%$ du résultat des enseignants.

Ces critiques sont accompagnées de la suspicion envers les mécanismes de recrutement et de promotion des enseignants. L'accès à l'enseignement se fait de façon automatique à travers les écoles normales du pays, que ces experts décrivent comme désuètes et de basse qualité, mais qui représentent une voie d'ascension sociale pour les jeunes des classes populaires. Si les jeunes accèdent à l'enseignement à travers les écoles normales, leur désignation définitive dépend du syndicat, dans un schéma paritaire ayant permis l'apparition d'un marché noir de vente de postes. La gestion des promotions se fait à travers un système de points récompensant l'ancienneté et les diplômes, des critères susceptibles de manipulation par les agents des commissions. Ces mécanismes sont critiqués comme la source des mauvais résultats, de l'inefficience et de la corruption du système éducatif national. Si ces critiques s'adressent au SNTE, ce sont les enseignants qui se voient affectés par le nouveau dispositif mis en place.

Élaborée par des experts mexicains travaillant étroitement avec l'OCDE${ }^{2}$, la réforme éducative de 2013 conçoit les enseignants comme la cible prioritaire de la politique éducative. Est ainsi introduit le Service professionnel enseignant, un système d'évaluations standardisées obligatoires supervisé par l'Institut national pour l'évaluation de l'éducation (INEE) ${ }^{3}$. Le nouveau dispositif comprend des concours d'accès et de promotion et des évaluations de la performance, que les enseignants doivent passer tous les quatre ans. Ces dernières, qui conditionnent la permanence des enseignants dans la fonction, sont les plus controversées et font face à de fortes mobilisations, menées par la CNTE dans la capitale et dans les États du sud du pays ${ }^{4}$.

\section{Un retour du corporatisme?}

6 La forte politisation de la question éducative au cours de la période 2012-2018 en a fait l'un des principaux éléments de débat pendant la dernière campagne présidentielle. Alors que la plupart des candidats défendent la réforme et ne proposent que des ajustements cosmétiques au dispositif d'évaluation, López Obrador, le candidat de la gauche, reprend les demandes syndicales d'abrogation et de restitution des enseignants licenciés. La fin de la réforme devient une évidence après la victoire de son parti, Morena, aux élections présidentielles et législatives, où plusieurs anciens cadres 
syndicaux sont élus comme parlementaires. Cela se traduit par un important travail législatif aboutissant en mai 2019 à la réforme de l'article 3 de la Constitution et à la dissolution de l'INEE et du SPD.

Défaire le SPD était un objectif central de la nouvelle politique éducative, qui vise à revaloriser le travail enseignant. Tandis que le gouvernement antérieur rendait les enseignants responsables de la qualité du système éducatif à travers un instrument contraignant d'accountability, l'administration d'Obrador adopte une approche formative de l'évaluation et délie les résultats des conséquences professionnelles, comme l'indique la Loi générale de la carrière des enseignantes et des enseignants, approuvée en septembre 2019. Cette loi nuance les concours « publics, transparents et en égalité de conditions » inscrits au texte constitutionnel en mai et donne priorité d'accès à la profession aux élèves des écoles normales et de l'Université pédagogique nationale. Par ailleurs, elle marque le retour de l'ancienneté ou des diplômes comme critères d'évaluation devant permettre aux enseignants d'accéder à des reconnaissances matérielles ou symboliques. Finalement, elle restitue le caractère paritaire des commissions devant décider des nouvelles entrées et promotions, où siègeront les administrations locale et fédérale et les syndicats.

8 Ainsi, cette loi répond aux demandes formulées par la CNTE au cours des dernières années, comme l'abrogation de la réforme de 2013, la fin des évaluations standardisées, le rétablissement d'une gestion paritaire ou la priorité donnée aux normaliens dans l'accès à la profession. Tout en déclarant vouloir éviter la reproduction des «vices corporatistes", le gouvernement rétablit les directions syndicales dans leur rôle d'interlocuteurs, dans un geste qui semble rechercher la stabilité politique du domaine éducatif. Ce rapprochement a été critiqué par l'opposition et par certains chercheurs en éducation ${ }^{5}$, qui y voient un retour aux principes d'organisation corporatistes et donc un potentiel foyer de corruption.

\section{L'austérité républicaine, quels enjeux pour la profession enseignante?}

9 Les instruments d'évaluation et leur opérationnalisation n'ayant pas encore été concrètement définis, il est encore tôt pour apprécier les implications que ces reconfigurations auront pour la profession. Cependant, un certain décalage semble exister entre la rhétorique et les actions du gouvernement. Alors que sont annoncés le rétablissement de la dignité du magistère et la revalorisation des écoles normales, les budgets de 2019 envoient des signaux contradictoires. La doctrine de l'«austérité républicaine " annoncée par le président pour lutter contre la corruption s'est traduite en pratique par une forte réduction des dépenses publiques. Ainsi, le budget pour la formation continue des enseignants est passé d'environ 20 millions d'euros en 2018 à 8,7 millions en 2019. Pour les écoles normales, la situation est encore plus dramatique : leur financement passe de 55,8 millions en 2016 à 12,6 millions en $2019^{6}$. Ces mesures ont provoqué la protestation du Congrès national des écoles normales, qui pour l'instant n'a eu d'écho ni dans les organisations syndicales ni au gouvernement.

Dans un article récent, l'écrivain Rafael Lemus considère que López Obrador est enfermé dans le " labyrinthe néolibéral » : alors que le président annonce la défaite du néolibéralisme, dans la pratique se cacherait, sous couvert de rupture, une forte 
continuité . Dans le domaine éducatif, si Obrador annonce simultanément la fin d'un dispositif d'évaluation considéré comme néolibéral et technocratique et le non-retour au corporatisme, le résultat est susceptible d'articuler le pire des deux mondes. C'est le «labyrinthe corporatiste ». D'un côté, la réconciliation des bureaucraties publique et syndicale pourrait accentuer des pratiques telles que le népotisme, la vente de postes ou leur distribution en échange de loyautés politiques. Ces pratiques n'ont par ailleurs pas disparu ces dernières années - on estime que, entre 2017 et 2018, seulement 25 à $30 \%$ des attributions de poste ont été réalisées à travers le $\mathrm{SPD}^{8}$. D'autre part, la réduction des fonds attribués à la formation initiale et continue des enseignants contribue à la précarisation d'une profession déjà fragilisée par quatre décennies de politiques néolibérales. Au cœur des ambigüités de ce labyrinthe demeurent donc aussi les enseignants, toujours enjoints d'agir comme agents de transformation sociale dans des conditions qui ne s'améliorent pas.

\section{NOTES}

1. La Commission économique pour l'Amérique latine et les Caraibes estime que les salaires réels enseignants ont chuté de $50 \%$ dans la région au cours des années 1980. CEPAL, Educación y conocimiento: eje de la transformación productiva con equidad, CEPAL, 1992, p. 216.

2. OCDE, Mejorar las escuelas: Estrategias para la acción en México, México, OCDE Publishing, 2010 ; OCDE, Establecimiento de un marco para la evaluación e incentivos docentes. Consideraciones para México, Paris, OECD Publishing, 2011.

3. Si l'INEE valide technique et juridiquement les instruments d'évaluation, leur élaboration dépend du secrétariat de l'éducation publique (SEP), qui a mandaté le Centre national d'évaluation de l'enseignement supérieur (Ceneval) pour cette tâche.

4. Norma Georgina Gutiérrez, « Mexique : tensions et utopies autour de la réforme dans les États d'Oaxaca et du Chiapas ", Revue internationale d'éducation de Sèvres, 2016, n 73, p. 15-19.

5. Carlos Ornelas, "Ascension et chute de la réforme éducative mexicaine: 2013-2019». Présentation au $\mathrm{IV}^{\mathrm{e}}$ colloque de la Revue internationale d'éducation de Sèvres. En ligne [http:// journals.openedition.org/ries/7322].

6. Les montants en pesos mexicains sont respectivement: 429,5 millions ; 187 millions ; 1200 millions ; 271 millions.

7. Rafael Lemus, «AMLO en el laberinto neoliberal », The New York Times, 8/07/2019.

8. Pablo Clark García-Dobarganes, « Ponencia en audiencia pública », Audiences publiques dans le domaine éducatif », Chambre des Députés, México, 13/02/2019. 
INDEX

Index géographique : Mexique

Mots-clés : évaluation de l'enseignant, politique éducative

Keywords : teacher evaluation, educational policy

Palabras claves : evaluación del docente, política educacional

\section{AUTEUR}

PABLO CUSSAC

Pablo Cussac est doctorant au Centre d'études européennes et de politique comparée (CEE) de Sciences Po, où il réalise, sous la direction de Philippe Bezes et d'Hélène Combes, une thèse sur les instruments utilisés pour évaluer les enseignants au Mexique et au Chili. Ses recherches, à la croisée de la sociologie des instruments, de l'administration et de l'évaluation, étudient les relations entre les groupes professionnels enseignants, l'administration et les experts éducatifs à la lumière des usages et des résistances à ces instruments. Courriel : pablo.cussac@sciencespo.fr 